\title{
Decline in serum progastrin-releasing peptide predicts the response of patients with small cell lung cancer to chemotherapy
}

\author{
MING LI ${ }^{1-4}$, DANDAN HAN ${ }^{3}$, WEI WANG ${ }^{1}$, YANG ZHANG $^{3}$, DAN $^{3} I^{3}$, \\ CHUNYANG DAI ${ }^{3}$, LITING QIAN $^{3}$ and WENCHU LIN ${ }^{1,4}$ \\ ${ }^{1}$ High Magnetic Field Laboratory, Chinese Academy of Sciences, Hefei, Anhui 230031; \\ ${ }^{2}$ University of Science and Technology of China, Hefei, Anhui 230036; \\ ${ }^{3}$ Department of Laboratory Diagnostics, The First Affiliated Hospital of University of Science and Technology of China; \\ ${ }^{4}$ Key Laboratory of High Magnetic Field and Ion Beam Physical Biology, Hefei Institutes of Physical Science, \\ Chinese Academy of Sciences, Hefei, Anhui 230031, P.R. China
}

Received March 6, 2020; Accepted August 18, 2020

DOI: $10.3892 / 01.2020 .12164$

\begin{abstract}
The utility of serum progastrin-releasing peptide (ProGRP) and neuron-specific enolase (NSE) as biomarkers for treatment monitoring and as prognostic factors was investigated in small cell lung cancer (SCLC) patients. Patients were first diagnosed pathologically at the First Affiliated Hospital of the University of Science and Technology of China and had their serum ProGRP and NSE levels measured using an electrochemiluminescence immunoassay. A total of 120 SCLC patients were enrolled. In responsive patients, ProGRP levels decreased significantly following two cycles of chemotherapy and continued to decline over the course of treatment. However, this decrease in ProGRP levels was not observed in non-responsive patients. Changes in ProGRP levels were more accurate than changes in NSE levels for monitoring the effects of chemotherapy in patients with SCLC. Following two treatment cycles or after the occurrence of drug resistance, changes in ProGRP levels in patients with low ProGRP levels at the time of diagnosis were not notably, regardless of whether or not patients were responders. The area under the receiver operating characteristic curve of the decline in ProGRP levels as a therapeutic biomarker of SCLC was 0.9643 , and the cut-off value was $55.02 \%$. A decline in ProGRP levels maybe a good predictor of objective response to chemotherapy in patients with SCLC with higher ProGRP levels at diagnosis. This model is expected to replace or be combined with imaging to predict chemotherapeutic treatment effects in patients with SCLC.
\end{abstract}

Correspondence to: Professor Wenchu Lin, High Magnetic Field Laboratory, Chinese Academy of Sciences, 350 Shushanhu Road, Hefei, Anhui 230031, P.R. China

E-mail: wenchu@hmfl.ac.cn

Key words: progastrin-releasing peptide, therapeutic monitoring, small-cell lung cancer, neuron-specific enolase

\section{Introduction}

Lung cancer is one of the most common malignancies and leads to the highest cancer-associated mortality rate in China (1). Approximately $10-15 \%$ of patients with lung cancer are diagnosed with small-cell lung cancer (SCLC), and $~ 70 \%$ of them are diagnosed at advanced stages (2). SCLC is a highly aggressive disease and has an increased tendency to metastasize. Patients with SCLC are generally treated with platinum-based chemotherapy alone or in combination with radiotherapy according to tumor stage. Although $60-80 \%$ of SCLC patients respond to first-line treatment, most of them inevitably develop chemoresistance and relapse within a relatively short time, leading to the spread of the disease $(3,4)$. Therapeutic efficacy is routinely evaluated based on imaging outcomes. However, imaging-observed changes in tumor volume are not notable in certain patients receiving effective treatment, but imaging cannot be performed prior to each chemotherapy cycle due to radiation exposure, and this may result in a failure to detect recurrence and/or metastasis in a timely manner. Therefore, there is an increasing requirement for convenient tools to detect responsiveness to treatment and predict prognosis in patients with SCLC in order to optimize disease management.

Several tumor markers have been used in patients with SCLC to improve diagnosis and treatment selection. Progastrin-releasing peptide (ProGRP) and neuron-specific enolase (NSE) are the most commonly used tumor markers in SCLC $(5,6)$. NSE has been the traditionally recommended tumor marker for SCLC. However, several studies have demonstrated that the diagnostic proficiency of ProGRP was higher than that of NSE in SCLC (7). McDonald et al first isolated gastrin-releasing peptide (GRP) from gastric nerve fibers in 1978 (8). In 1988, immunohistochemical studies confirmed the presence of GRP and its peptide precursor in SCLC cell lines. Since then, several studies have investigated the potential use of GRP as a biomarker in SCLC; however, this was found to be challenging because GRP was unstable in plasma and therefore, GRP levels were difficult to measure accurately. ProGRP is the precursor of GRP and is more 
stable than GRP in plasma. Circulating ProGRP has been demonstrated to be an effective biomarker for discriminating SCLC from non-small cell lung cancer and benign lung diseases with high sensitivity and specificity $(9,10)$. Numerous studies have focused on serum ProGRP and NSE as tumor markers for diagnosing patients with SCLC. However, few studies have prospectively evaluated the use of ProGRP and NSE levels as therapeutic and prognostic indicators in patients with SCLC (11), particularly in patients with SCLC with higher ProGRP levels at diagnosis. In the present study, the changes of the ProGRP levels in the responder group and non-responder group were observed, and whether changes in ProGRP levels may predict treatment response for SCLC was investigated.

\section{Materials and methods}

Patients. Data were retrospectively collected regarding 285 patients with SCLC who were initially diagnosed at The First Affiliated Hospital of the University of Science and Technology of China between January 2015 and October 2018, and complete information was available for 120 cases. A diagnosis of SCLC was made pathologically using bronchoscopic biopsies, CT-guided needle lung biopsies, or surgically resected specimens. The following inclusion criteria were used: i) Diagnosis was confirmed pathologically; ii) patients were first diagnosed prior to receiving any treatment; iii) NSE and ProGRP levels were measured prior to treatment; iv) NSE and ProGRP levels were measured at several time points throughout the treatment period; v) the entire chemotherapy process was performed in the hospital, with a 6-month follow-up time; and vi) indicators associated with renal function, including creatinine, creatinine clearance rate, and urine nitrogen were at normal levels. Based on Response Evaluation Criteria in Solid Tumors version 1.1, the treatment response was divided into responder and non-responder groups (12). The responder group included patients who had a partial response (PR) and those who had a complete response (CR), and the non-responder group included patients who had stable disease (SD) and those who had progressive disease (PD). Chemoresistance was defined as progression or relapse following first-line treatment in patients with SCLC. ProGRP and NSE levels, and imaging outcomes were evaluated retrospectively at the time of diagnosis following two cycles of chemotherapy and post-chemoresistance. The present study was approved by the Ethics Committee of The First Affiliated Hospital of the University of Science and Technology of China. Due to the retrospective nature of this study, written informed consent was not required.

ProGRP and NSE assay. Serum ProGRP and NSE levels were determined using an electrochemiluminescence immunoassay (ECLIA) on a Cobas E601 Analyzer system (Roche Applied Science). All specimens were processed within $6 \mathrm{~h}$ of collection using a centrifugal protocol $(2,200 \mathrm{x}$ g for $10 \mathrm{~min}$ at room temperature). The serum ProGRP sensitivity cut-off level was $75.3 \mathrm{pg} / \mathrm{ml}$, which was the same as that stated in the Roche reagent protocol (13). A high level of ProGRP was defined as the level of ProGRP above the normal reference range, and a low level of ProGRP was defined as a value within the normal reference range.

Statistical analysis. All the statistical analyses were performed using GraphPad Prism version 8.5 (GraphPad Software, Inc.) and SPSS version 19.0 (IBM Corp.). All data are presented as the median and quartiles $(\mathrm{Q}, 25$ and 75 th percentiles), and statistical analysis was performed using Friedman's test, followed by the Nemenyi post-hoc test. Receiver operating characteristic (ROC) curves were used to display the correlation between sensitivity and specificity, and the therapeutic efficacy was assessed by calculating the area under curve (AUC). Survival rate curves were drawn using the Kaplan-Meier method, and the log-rank test was used to compare the differences in the curves. Changes in ProGRP and NSE levels following chemotherapy were performed using the Wilcoxon signed-rank test. $\mathrm{P}<0.05$ was considered to indicate a statistically significant difference.

\section{Results}

Baseline clinicopathological features of patients with SCLC. Of the total 285 patients with SCLC, 58 (20.4\%) had limited disease (LD) and 227 (79.6\%) had extensive disease (ED), and $26.6 \%(68 / 256)$ of patients had a low level of ProGRP and74.4\% (188/256) had a high level of ProGRP. A total of $80 \%(228 / 285)$ of the total 285 patients with SCLC were male (Table SI). Among the 120 patients with SCLC, the median age was 64 years (range, 43-85 years). Ninety-nine $(82.5 \%)$ patients were male and $21(17.5 \%)$ were female. Seventy patients were non-smokers and 50 were current or ex-smokers. Twenty-three patients had LD at the time of diagnosis, and the other 97 patients had ED. The median level of ProGRP in patients with $\mathrm{LD}$ was $589 \mathrm{pg} / \mathrm{ml}(\mathrm{Q}, 55.74-2,263.00 \mathrm{pg} / \mathrm{ml})$, and the median level of ProGRP in patients with ED was $1,742 \mathrm{pg} / \mathrm{ml}(\mathrm{Q}, 137.2-4,006.5 \mathrm{pg} / \mathrm{ml}$; Table I).

ProGRP as a therapeutic biomarker. Changes in ProGRP and NSE levels in the 120 patients with SCLC were analyzed, including at diagnosis, following two cycles of chemotherapy and following the occurrence of drug resistance, and levels of serum ProGRP and NSE were also measured prior to the start of chemotherapy. The highest point of detection of ProGRP is $5,000 \mathrm{pg} / \mathrm{ml}$, and results $>5,000 \mathrm{pg} / \mathrm{ml}$ were reported as $5,000 \mathrm{pg} / \mathrm{ml}$. Levels of serum ProGRP and NSE significantly decreased following two cycles of chemotherapy $(\mathrm{P}<0.001)$ and significantly increased following drug resistance $(\mathrm{P}<0.001$; Fig. 1). Figs. 2 and 3 are the breakdown data of Fig. 1. There were 88 responders and 32 non-responders. In the responders, serum ProGRP levels following two cycles of chemotherapy were significantly lower than baseline levels at diagnosis ( $\mathrm{P}<0.001$; Fig. 2A). In non-responders, following two cycles of chemotherapy, there were no significant decreases in serum ProGRP concentrations (Fig. 2B; $\mathrm{P}=0.752$ ). This was also the case for the concentrations of NSE in patients with SCLC (Fig. 3).

Decline in ProGRP levels predicts objective response to treatment. The association between decreases in serum ProGRP and NSE levels and the effectiveness of SCLC chemotherapy 
Table I. Baseline characteristics of patients with small-cell lung cancer.

\begin{tabular}{lcccc}
\hline Characteristic & $\mathrm{n}(\%)$ & ProGRP, pg/ml $(95 \% \mathrm{CI})$ & P-value & NSE, ng/ml (95\% CI) \\
\hline Sex & & & & \\
$\quad$ Male & $99(82.5)$ & $1,428(70.3-3568)$ & 0.346 & $41.37(19.98-69.5)$ \\
Female & $21(17.5)$ & $2,583(154.6-4901.5)$ & & $43.92(25.96-113.45)$ \\
Age & & & & \\
$<64$ & $58(48.3)$ & $2,064(351.35-3580.75)$ & 0.168 & $45.46(22.13-79.55)$ \\
$\geq 64$ & $62(51.7)$ & $775.3(59.15-3870.25)$ & & $35.8(20.60-67.20)$ \\
Smoking & & & & 0.652 \\
Smoker & $50(41.7)$ & $1,338.5(95.67-3407)$ & 0.548 & $39.12(18.93-67.20)$ \\
Non-smoker & $70(58.3)$ & $1,608(67.28-4458.25)$ & & $46.29(23.16-80.10)$ \\
Stage & & & & 0.259 \\
Limited & $23(19.2)$ & $589(55.74-2263)$ & 0.092 & $27.95(19.24-58.14)$ \\
Extensive & $97(80.8)$ & $1,742(137.2-4006.5)$ & & $46.44(23.20-76.38)$
\end{tabular}

ProGRP, progastrin releasing peptide; NSE, Neuron-specific enolase; CI, confidence interval.
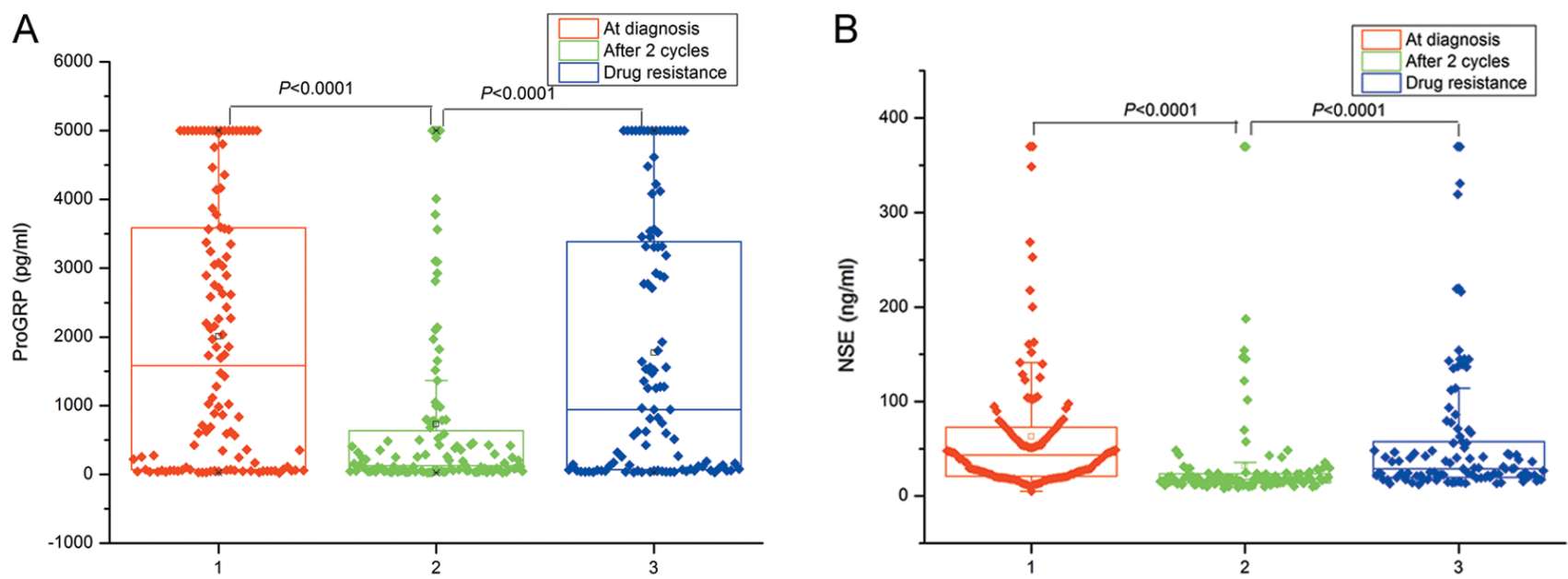

Figure 1. Changes in ProGRP and NSE levels in the 120 patients with SCLC using non-parametric tests. (A) Changes in ProGRP levels in SCLC; (B) changes in NSE levels in SCLC. ProGRP, progastrin-releasing peptide; NSE, Neuron-specific enolase; SCLC, small-cell lung cancer.

are shown in Fig. 4. Serum ProGRP levels were associated with greater diagnostic accuracy and were predictive of a patient's objective response to SCLC chemotherapy; corresponding ROC curves showed a rate of ProGRP decline of 0.8572 (cut-off, 55.02\%; $\mathrm{P}<0.001$ ).

Notably, it was reported that following two cycles of treatment or drug resistance, further changes in ProGRP levels in patients with low ProGRP levels at the time of diagnosis were not notable (Fig. 5), regardless of whether patients were responders or non-responders. The cut-off level of serum ProGRP was $75.3 \mathrm{pg} / \mathrm{ml}$, as specified by the reagent manufacturer. The AUC for the decline in higher ProGRP levels as a biomarker for treatment monitoring of SCLC was 0.9643 $(\mathrm{P}<0.001)$. A decline in ProGRP levels may be used as a good predictor of objective response to SCLC chemotherapy, and the cut-off value also was $55.02 \%$ (Fig. 6).

To verify whether changes in ProGRP levels may be used to assess the efficacy of SCLC chemotherapy, the association between expression levels of ProGRP and NSE, as well as imaging characteristics of solid tumors, was investigated. Patients with SCLC were diagnosed at an advanced disease stage and were treated with cisplatin combined with etoposide. From the imaging data, tumor shrinkage was observed and the treatment response was assessed as PR. Levels of ProGRP declined steadily and synchronously (Fig. 7); however, tumor size began to increase again following six cycles of chemotherapy. In line with the changes in tumor radiological characteristics, ProGRP levels steadily decreased from the start of treatment until five cycles of chemotherapy had been completed and then increased following six cycles of chemotherapy. Although NSE expression also significantly decreased from the start of treatment until five cycles of chemotherapy, it did not increase after six cycles of chemotherapy. These data indicated that serum ProGRP maybe used as a potential biomarker for monitoring the therapeutic response in patients with SCLC. 

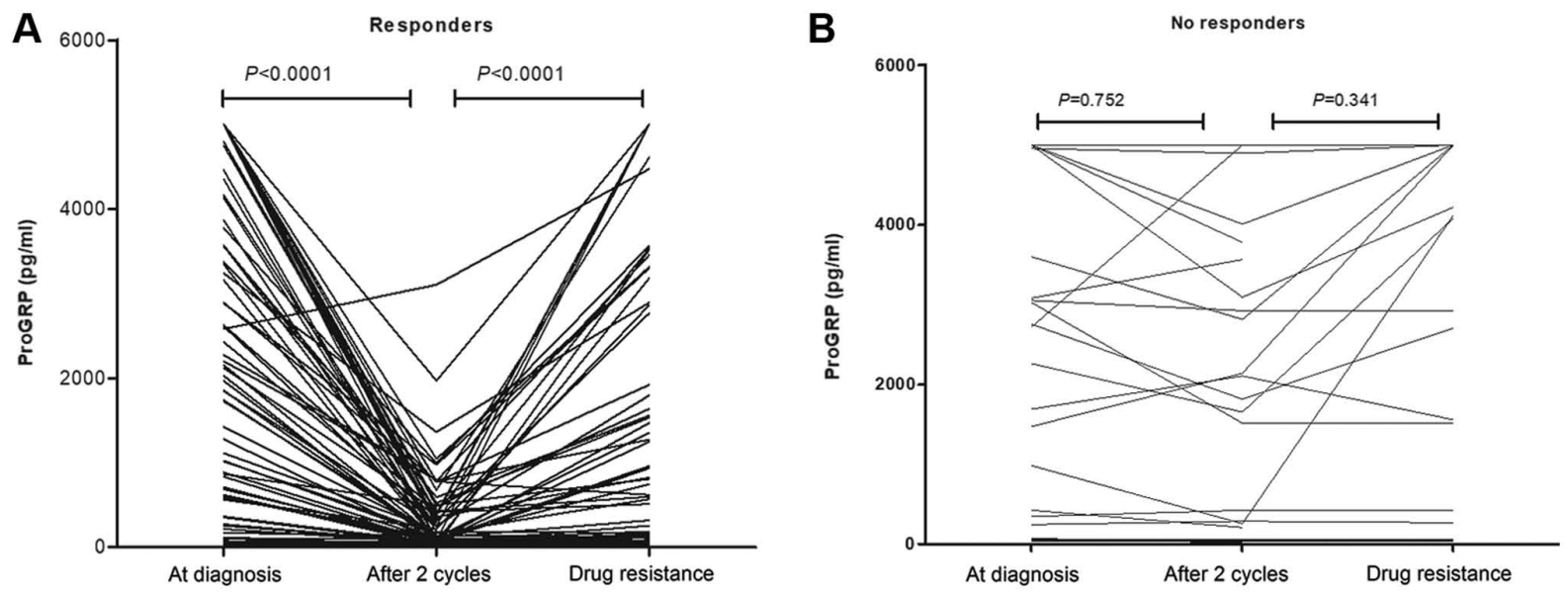

Figure 2. Changes in ProGRP levels in the 120 patients with SCLC prior to and following treatment using the Mann-Whitney U test for (A) 88 responders and (B) 32 non-responders. ProGRP, progastrin releasing peptide; SCLC, small-cell lung cancer.

A

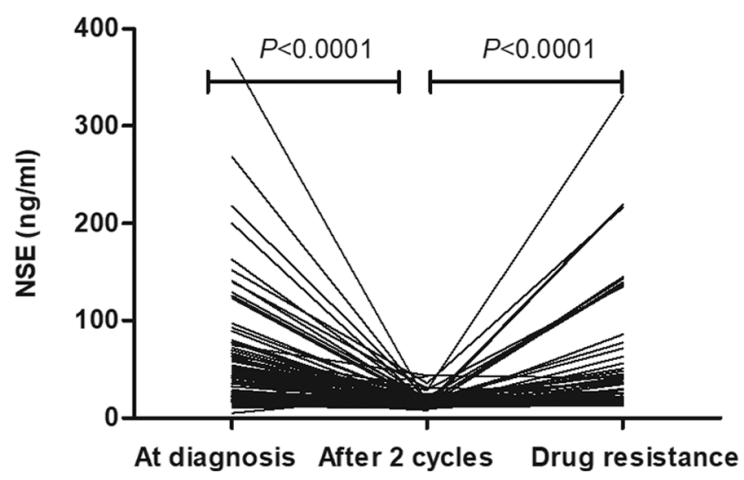

B

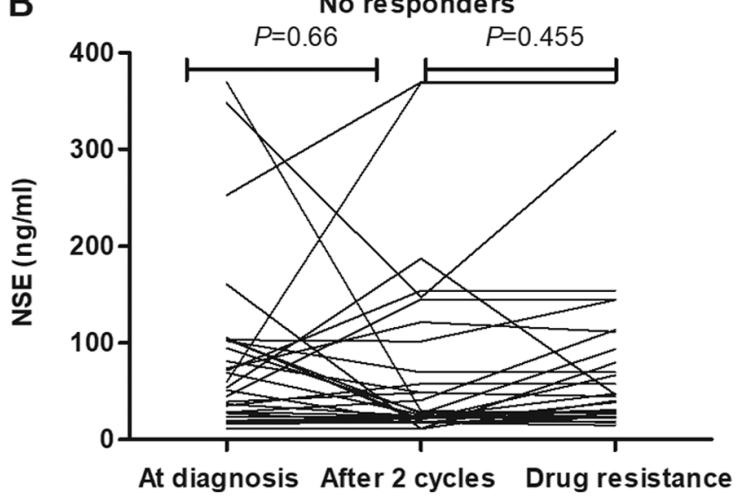

Figure 3. Changes in NSE levels in the 120 patients with SCLC prior to and following treatment using the Mann-Whitney U test for (A) 88 responders and (B) 32 non-responders. NSE, Neuron-specific enolase; SCLC, small-cell lung cancer.
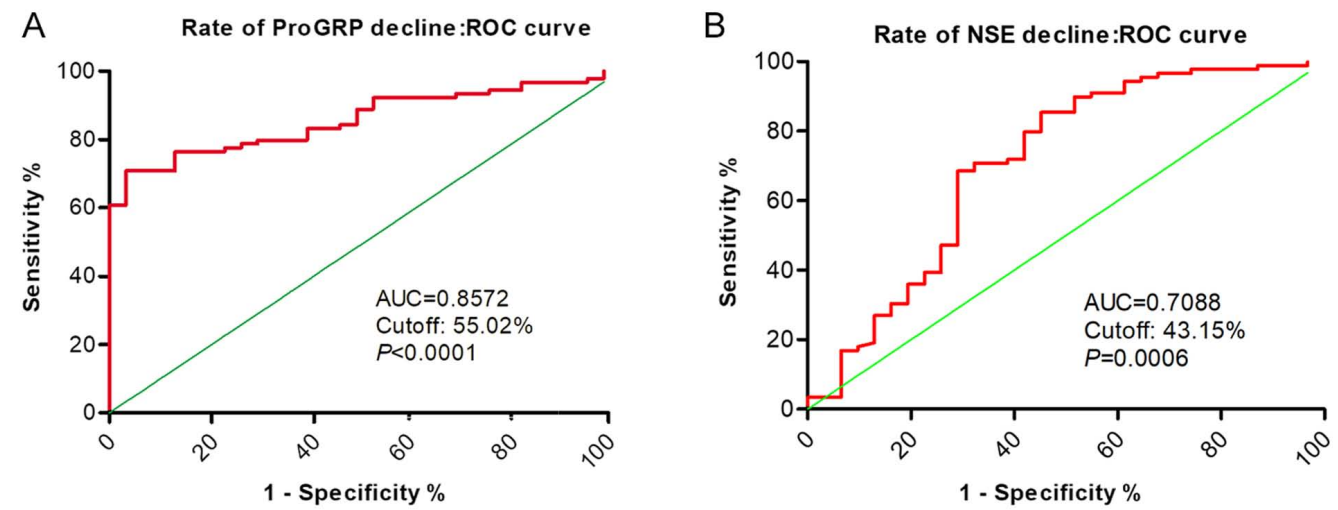

Figure 4. ROC curves of rate of ProGRP and NSE decline on the therapeutic biomarker of SCLC. (A) ProGRP decline level in SCLC; (B) NSE decline level in SCLC. ROC, receiver operating characteristic; ProGRP, progastrin-releasing peptide; NSE, Neuron-specific enolase; SCLC, small-cell lung cancer.

Survival and prognosis according to ProGRP and NSE. Among the 120 patients with SCLC, median progression-free survival (PFS) times were significantly shorter in patients with SCLC with ED ( $\mathrm{P}=0.006)$ and higher ProGRP $(\mathrm{P}=0.048)$ and NSE $(\mathrm{P}=0.001)$ levels. However, no significant differences in PFS were identified when comparing male and female patients $(\mathrm{P}=0.904)$, older and younger patients $(\mathrm{P}=0.276)$, or smokers and non-smokers $(\mathrm{P}=0.669)$. In multivariate analysis, the level of NSE [95\% CI: 0.567 (0.384-0.837); $\mathrm{P}=0.004$ ] and disease stage [95\% CI: 0.543 (0.322-0.915); $\mathrm{P}=0.022$ ] were prognostic factors of survival in patients with SCLC (Table II). The median PFS time was significantly longer in patients with lower levels of baseline serum NSE, according to Kaplan-Meier survival curves (Fig. 8). 

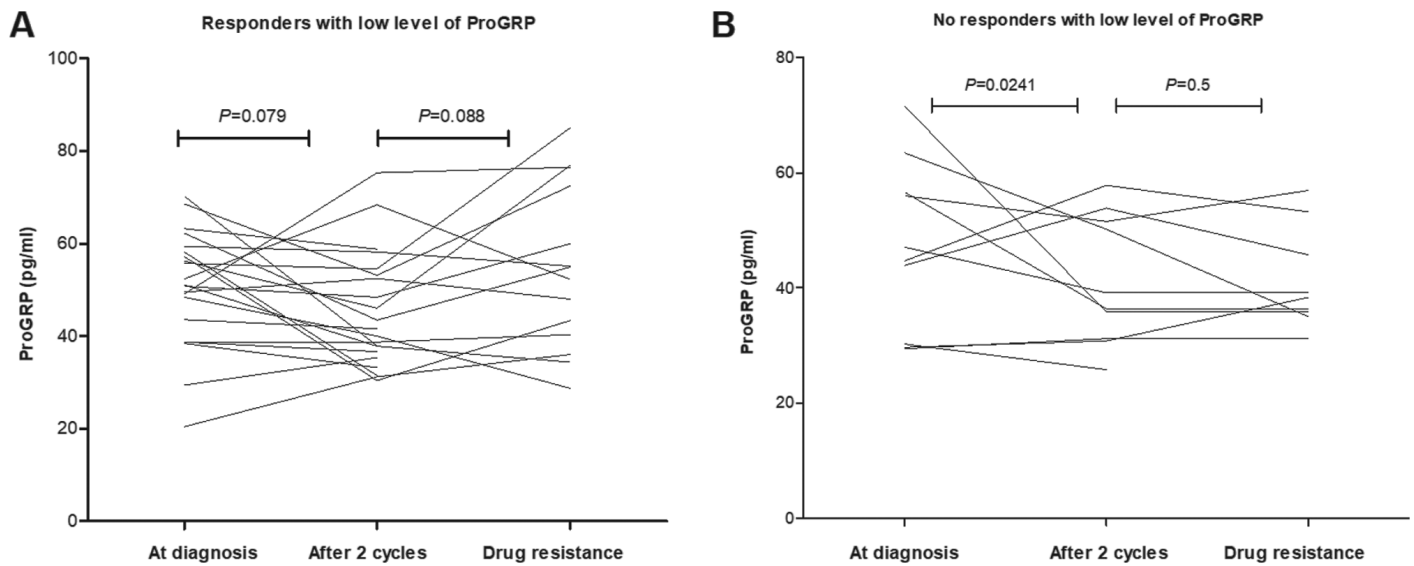

Figure 5. Changes of 32 SCLC patients with low-value ProGRP levels at the time of diagnosis prior to and following treatment. (A) Responders. (B) Non-responders. ProGRP, progastrin-releasing peptide; SCLC, small-cell lung cancer.

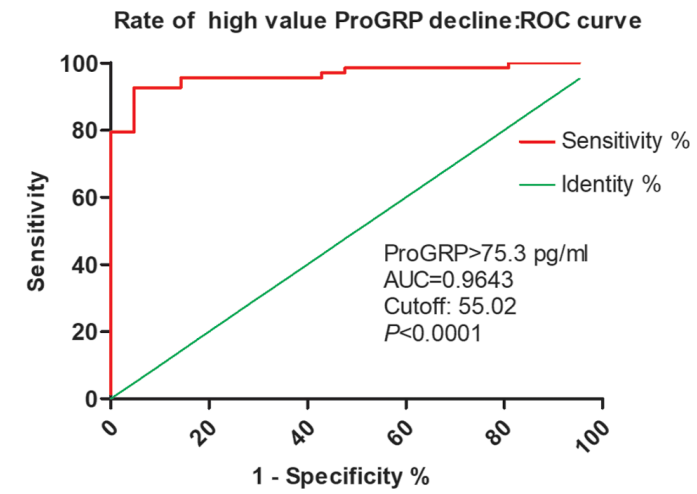

Figure 6. ROC curves associated with higher ProGRP levels that showed a decline in the therapeutic biomarker of SCLC (AUC, 0.9643; P<0.001). ROC, receiver operating characteristic; ProGRP, progastrin-releasing peptide; SCLC, small-cell lung cancer.

\section{Discussion}

One of the main treatment methods for SCLC is chemotherapy, and first-line treatment for SCLC is cisplatin combined with etoposide. The efficacy of chemotherapy is routinely evaluated based on imaging following two cycles of chemotherapy. Therefore, ProGRP and NSE serum concentrations and imaging outcome data at the time of diagnosis, after two cycles of chemotherapy, and following drug resistance, were obtained retrospectively. Previous studies have reported that serum ProGRP and NSE levels may provide useful diagnostic and prognosis value in SCLC $(14,15)$. However, due to the lack of large-scale clinical trial data, the role of ProGRP and NSE as biomarkers of treatment efficacy remains controversial (16). In the present study, information was collected regarding 285 patients with SCLC who were hospitalized in The First Affiliated Hospital of University of Science and Technology of China, and 120 patients with complete information were analyzed. The clinical characteristics of the patients were consistent with those of previous studies $(17,18)$. The results of the present study demonstrated that changes in serum ProGRP levels may be used as a biomarker to monitor therapeutic efficacy in patients with SCLC. Among the 120 patients with SCLC who were followed, levels of serum ProGRP in responders decreased significantly following chemotherapy, while there was no significant decrease in the concentration of serum ProGRP in non-responders when comparing pre- and post-treatment levels. Ono et al reported that a decline in serum ProGRP levels was strongly correlated with tumor diameter shrinkage prior to the third course of treatment (19). Changes in serum ProGRP levels showed better correlation with overall tumor diameter shrinkage than did changes in serum NSE levels. Therefore, the results demonstrated that changes in ProGRP, compared with NSE, were more reliable for monitoring treatment and predicting relapse in patients with SCLC.

Notably, the present study reported that $26.6 \%$ of patients with SCLC had a low level of ProGRP at diagnosis and had smaller changes in ProGRP levels following chemotherapy or recurrence, regardless of whether patients were responders or non-responders, which may be associated with lower GRP expression levels in these SCLC patients. Wojcik and Kulpa reported that $\sim 30 \%$ of patients with SCLC had low GRP expression levels (20), indicating that ProGRP levels would not be suitable as a clinical biomarker for this group of patients. Numerous studies have focused on serum proGRP as a diagnostic marker for patients with SCLC. However, few studies have systematically assessed the role of proGRP levels in monitoring treatment efficacy in patients with $\operatorname{SCLC}(21,22)$. For patients with SCLC with high ProGRP levels at diagnosis, a model for predicting objective response to chemotherapy was established based on chemotherapy-related decreased levels in serum ProGRP. The AUC for the decline in higher ProGRP levels as a therapeutic biomarker of SCLC was 0.9643 , and the cut-off value was 55.02\%. Decreases in ProGRP levels may be a good predictor of the therapeutic response to SCLC chemotherapy. This predictive model may be used to replace imaging or be combined with imaging to evaluate treatment efficacy in patients with SCLC.

In addition, SCLC patient survival and prognosis were analyzed in the present study, and concentrations of NSE showed a clear association with prognosis in patients with SCLCs, which was weaker for ProGRP. We hypothesized that most of the SCLC patients with ProGRP levels in the normal range had low GRP expression levels, which may be a specific subtype of patients, who may be more likely to develop resistance to chemotherapy. Furthermore, in 
Table II. Univariate and multivariate Cox proportional hazard models for survival.

\begin{tabular}{lccc}
\hline Variable & Univariate analysis HR $(95 \% \mathrm{CI})$ & P-value & Multivariate analysis HR (95\% CI) \\
\hline Sex, male vs. female & $0.967(0.565-1.657)$ & 0.904 & \\
Age, <64 vs. $\geq 64$ & $0.806(0.546-1.188)$ & 0.276 & \\
Smoking, yes vs. no & $0.918(0.620-1.359)$ & 0.669 & \\
Stage, LD vs. ED & $0.483(0.287-0.815)$ & 0.006 & $0.543(0.322-0.915)$ \\
ProGRP, high vs. low & $0.664(0.443-0.996)$ & 0.048 & $0.714(0.475-1.073)$ \\
NSE, high vs. low & $0.530(0.360-0.781)$ & 0.001 & $0.567(0.384-0.837)$ \\
\hline
\end{tabular}

HR, hazards ratio; CI, confidence interval; ProGRP, progastrin releasing peptide; NSE, Neuron-specific enolase; LD, limited disease; ED, extensive disease.
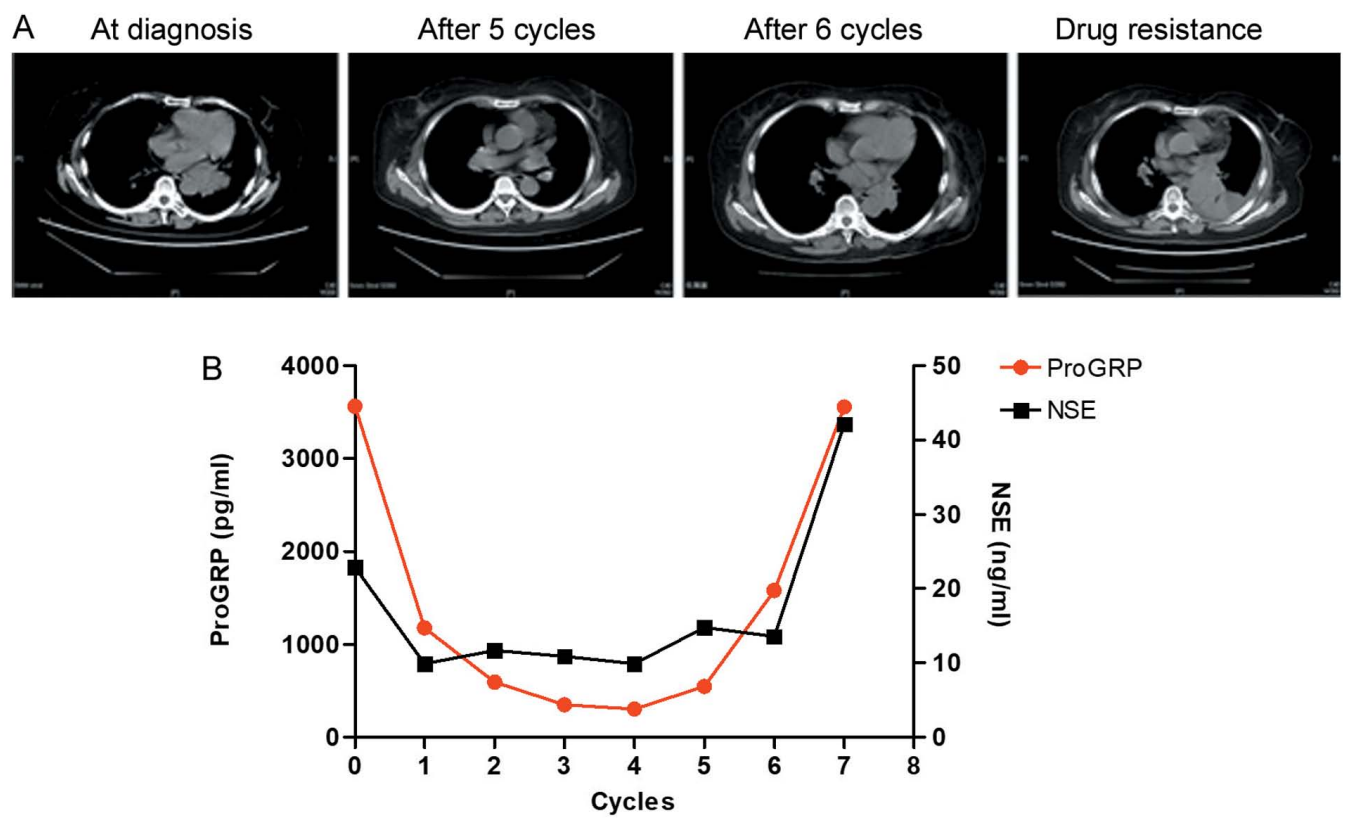

Figure 7. Dynamic change inpatient imaging characteristics and ProGRP and NSE levels. (A) Imaging characteristics; (B) ProGRP and NSE levels. ProGRP, progastrin releasing peptide; NSE, Neuron-specific enolase.
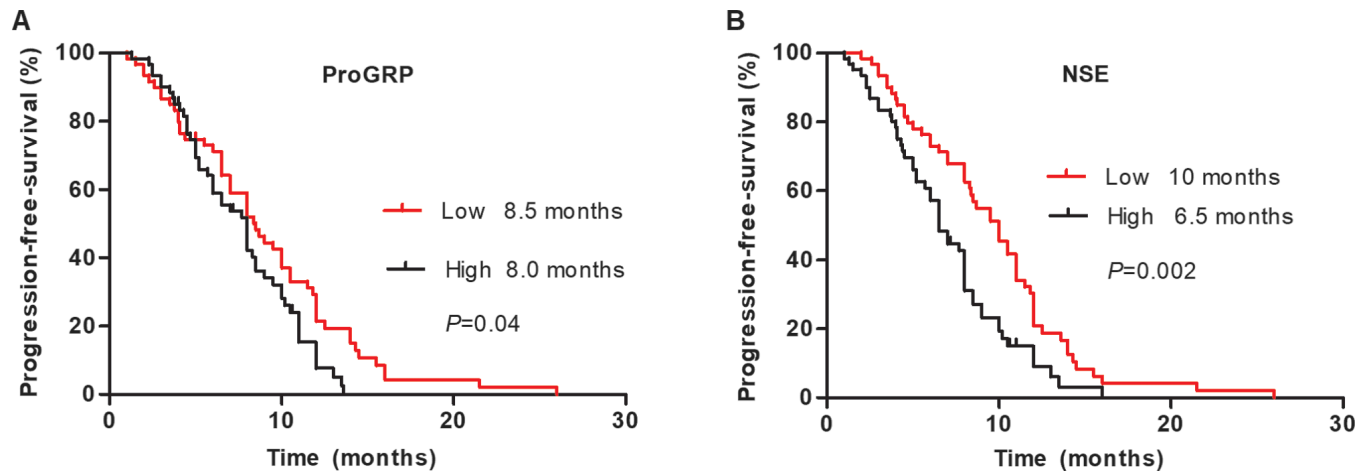

Figure 8. Kaplan-Meier survival curves of progression-free-survival based on baseline serum NSE and ProGRP levels. (A) ProGRP and (B) NSE levels in SCLC. ProGRP, progastrin releasing peptide; NSE, Neuron-specific enolase; SCLC, small-cell lung cancer.

multivariate analysis that included simultaneous evaluations of clinical parameters and concentrations of the two markers, NSE remained an independent predictor of survival (23).
In conclusion, changes in serum levels of ProGRP, which are initially high at the time of diagnosis, may aid in predicting the effectiveness of different chemotherapy regimens in patients with SCLC. There are certain limitations to the 
present study. The relatively small sample size may limit the generalization of the conclusions made, which will require further validation in larger cohorts. Further research will be required to determine whether there is cellular heterogeneity in patients with SCLC s who are either ProGRP-negative or -positive, and whether ProGRP-negative patients should be considered a specific subtype of SCLC.

\section{Acknowledgements}

Not applicable.

\section{Funding}

The present study was funded by the Fundamental Research Funds for the Central University (grant no. WK9110000025), the Natural Science Foundation of Anhui Province (grant no. 2008085MH288), the Science and Technology Major Project of Anhui Province (grant no. 18030801140), the National Natural Science Foundation of China (grant no. 81672647), the National Ministry of Industry and Information Technology Science and Technology (grant no. 2018MND102041).

\section{Availability of data and materials}

All data used and/or analyzed in the present study are available from the corresponding author upon reasonable request.

\section{Authors' contributions}

ML and WL contributed toward designing, drafting and revising the manuscript. DH, YZ, DL and CD and LQ contributed toward the collection and collation of clinical data. WW performed the statistics on clinical data. All authors read and approved the final manuscript.

\section{Ethics approval and consent to participate}

The present study was approved by the Ethics Committee of The First Affiliated Hospital of University of Science and Technology of China. Due to the retrospective nature of this study, written informed consent was not required.

\section{Patient consent for publication}

Not applicable.

\section{Competing interests}

All authors declare no competing interests regarding the present study.

\section{References}

1. Chen W, Zheng R, Baade PD, Zhang S, Zeng H, Bray F, Jemal A, Yue XQ and He J: Cancer statistics in China, 2015. CA Cancer J Clin 66: 115-132, 2016.

2. Kalemkerian GP, Akerley W, Bogner P, Borghaei H, Chow LQ, Downey RJ, Gandhi L, Ganti AK, Govindan R, Grecula JC, et al: Small cell lung cancer. J Natl Compr Canc Netw 11: 78-98, 2013.
3. Byers LA and Rudin CM: Small cell lung cancer: Where do we go from here? Cancer 121: 664-672, 2015.

4. Govindan R, Page N, Morgensztern D, Read W, Tierney R, Vlahiotis A, Spitznagel EL and Piccirillo J: Changing epidemiology of small-cell lung cancer in the United States over the last 30 years: Analysis of the surveillance, epidemiologic, and end results database. J Clin Oncol 24: 4539-4544, 2006.

5. Du J, Li Y, Wang L, Zhou Y, Shen Y, Xu F and Chen Y: Selective application of neuroendocrine markers in the diagnosis and treatment of small cell lung cancer. Clin Chim Acta 509: 295-303, 2020.

6. Wójcik E, Kulpa JK, Sas-Korczyńska B, Korzeniowski S and Jakubowicz J: ProGRP and NSE in therapy monitoring in patients with small cell lung cancer. Anticancer Res 28 (5B): 3027-3033, 2008.

7. Wu XY, Hu YB, Li HJ, Wan B, Zhang CX, Zhang B, Hu H, Zhang Q Lv TF, Zhan P and Song Y: Diagnostic and therapeutic value of progastrin-releasing peptide on small-cell lung cancer: A single-center experience in China. J Cell Mol Med 22: 4328-4334, 2018.

8. McDonald TJ, Nilsson G, Vagne M, Ghatei M, Bloom SR and Mutt V: A gastrin releasing peptide from porcine nonantral gastric tissue. Gut 19: 767-774, 1978.

9. Stieber P, Dienemann H, Schalhorn A, Schmitt MU, Reinmiedl J, Hofmann K and Yamaguchi K: Pro-gastrin-releasing peptide (ProGRP)-a useful marker in small cell lung carcinomas. Anticancer Res 19: 2673-2678, 1999.

10. Wang $\mathrm{H}$ and Qian J: Serum pro-gastrin-releasing peptide in diagnosis of small cell lung cancer: A meta-analysis. J Cancer Res Ther 12 (Suppl)2: C260-C263, 2016.

11. Huang Z, Xu D, Zhang F, Ying Y and Song L: Pro-gastrin-releasing peptide and neuron-specific enolase: Useful predictors of response to chemotherapy and survival in patients with small cell lung cancer. Clin Transl Oncol 18: 1019-1025, 2016.

12. Eisenhauer EA, Therasse P, Bogaerts J, Schwartz LH, Sargent D, Ford R, Dancey J, Arbuck S, Gwyther S, Mooney M, et al: New response evaluation criteria in solid tumours: Revised RECIST guideline (version 1.1). Eur J Cancer 45: 228-247, 2009.

13. Nordlund MS, Bjerner J, Warren DJ, Nustad K and Paus E: Progastrin-releasing peptide: Stability in plasma/serum and upper reference limit. Tumour Biol 29: 204-210, 2008.

14. Yang DW, Zhang Y, Hong QY, Hu J, Li C, Pan BS, Wang Q, Ding FH, Ou JX, Liu FL, et al: Role of a serum-based biomarker panel in the early diagnosis of lung cancer for a cohort of high-risk patients. Cancer 121 (Suppl 17): S3113-S3121, 2015.

15. Lv ShP, Wang Y, Huang L, Wang F, Zhou JG and Ma H: Meta-analysis of serum gastrin-releasing peptide precursor as a biomarker for diagnosis of small cell lung cancer. Asian Pac J Cancer Prev 18: 391-397, 2017.

16. Cavalieri S, Morelli D, Martinetti A, Galli G, Nichetti F, de Braud $\mathrm{F}$ and Platania M: Clinical implications for pro-GRP in small cell lung cancer. A single center experience. Int J Biol Markers 33: 55-61, 2018.

17. Oh HJ, Park HY, Kim KH, Park CK, Shin HJ, Lim JH, Kwon YS, Oh IJ, Kim YI, Lim SC, et al: Progastrin-releasing peptide as a diagnostic and therapeutic biomarker of small cell lung cancer. J Thorac Dis 8: 2530-2537, 2016.

18. Liu D, Huang Y, Li L, Song J, Zhang L and Li W: High neutrophil-to-lymphocyte ratios confer poor prognoses in patients with small cell lung cancer. BMC Cancer 17: 882, 2017.

19. Ono A, Naito T, Ito I, Watanabe R, Shukuya T, Kenmotsu H, Tsuya A, Nakamura Y, Murakami H, Kaira K, et al: Correlations between serial pro-gastrin-releasing peptide and neuron-specific enolase levels, and the radiological response to treatment and survival of patients with small-cell lung cancer. Lung Cancer 76: 439-444, 2012.

20. Wojcik E and Kulpa JK: Pro-gastrin-releasing peptide (ProGRP) as a biomarker in small-cell lung cancer diagnosis, monitoring and evaluation of treatment response. Lung Cancer (Auckl) 8: 231-240, 2017.

21. Holdenrieder S, von Pawel J, Dankelmann E, Duell T, Faderl B, Markus A, Siakavara M, Wagner H, Feldmann K, Hoffmann H, et al: Nucleosomes, ProGRP, NSE, CYFRA 21-1, and CEA in monitoring first-line chemotherapy of small cell lung cancer. Clin Cancer Res 14: 7813-7821, 2008.

22. Nisman B, Biran H, Ramu N, Heching N, Barak V and Peretz T: The diagnostic and prognostic value of ProGRP in lung cancer. Anticancer Res 29: 4827-4832, 2009.

23. Zhou M, Wang Z, Yao Y, Zhou H, Liu M and Sun J: Neuron-specific enolase and response to initial therapy are important prognostic factors in patients with small cell lung cancer. Clin Trans Oncol 19: 865-873, 2017. 